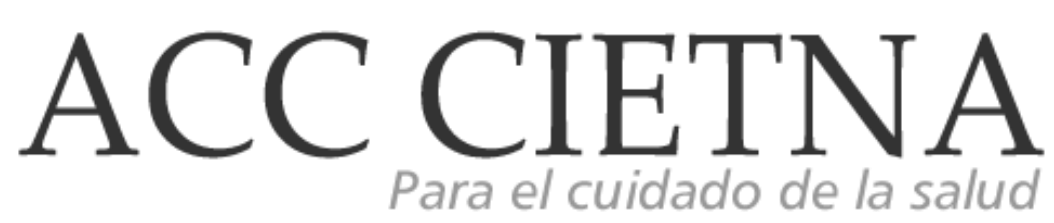

https://doi.org/10.35383/cietna.v4i2.16

\title{
Nivel de incertidumbre del familiar frente a la enfermedad, Unidad de Shock trauma - Hospital Nacional Almanzor Aguinaga Asenjo 2014
}

\section{Bernuy De los Ríos María Berenice ${ }^{1}$; Uriarte Uriarte Stefany Analiz²; Mesta Delgado Rosío del Pilar $^{3}$}

\begin{tabular}{l} 
INFORMACIÓN DEL ARTÍCULO \\
\hline Historia del artículo: \\
Recibido el 23 de mayo de 2017 \\
Aceptado el 15 de noviembre de 2017
\end{tabular}

\section{Palabras claves:}

Incertidumbre

Familiar

Unidad Shock Trauma

\section{RESUMEN}

Actualmente los servicios de Emergencia de los diferentes hospitales del Perú, se ven muy congestionados de pacientes, quienes presentan innumerables problemas de salud, muchos de ellos con compromiso hemodinámico. Debido a la inestabilidad de las personas afectadas, la enfermera centra su atención en el cuidado que debe brindar, dejando a un segundo plano la familia; originándoles una percepción de amenaza y experimentando una gran incertidumbre. La presente investigación tuvo como objetivo determinar el nivel de incertidumbre en el familiar frente a la enfermedad en la unidad de Shock Trauma, con el fin de contribuir a la reflexión profesional de las enfermeras, comprender las diferentes situaciones que el familiar enfrenta y el grado de incertidumbre que afrontan durante este periodo. Esta investigación utilizó el enfoque cuantitativo, descriptivo y de corte trasversal. Se tuvo una población de 634 y una muestra de 239 personas. En la investigación se utilizó como instrumento de recolección de datos una encuesta que toma como referencia la Escala de Incertidumbre de Mishel, se empleó la técnica de muestreo no probabilístico consecutivo, obteniendo como resultados que el $68 \%$ de

\footnotetext{
1 Licenciada. Enfermera con trabajo independiente, Chiclayo, Perú. Email: bernuydlrios@gmail.com

2 Licenciada. Enfermera con trabajo independiente, Chiclayo, Perú. Email: stefanyuriarte21@gmail.com

3 Magister en Enfermería. Docente de la Escuela de Enfermería de la Universidad Católica Santo Toribio de Mogrovejo, Chiclayo, Perú. Email: rmesta@usat.edu.pe
} 
los familiares tenía un nivel regular de incertidumbre, $26 \%$ de población tenía un nivel bajo de incertidumbre, y el $6 \%$ un nivel alto de incertidumbre. Por último se concluyó que a menor grado de instrucción y menor tiempo de permanencia genera mayor índice de incertidumbre en el familiar frente a la enfermedad.

Level of uncertainty of illness in the familiar, Shock trauma Unit Hospital Nacional Almanzor Aguinaga Asenjo 2014

ABSTRACT

\section{Keywords:}

Uncertainty

Family

Unit Shock Trauma
At present, the emergency services of the different hospitals in Peru are very congested of patients, who present innumerable health problems, many of them with hemodynamic compromise. Due to the instability of the affected people, the nurse focuses on the care she should provide, leaving the family to the background; Giving them a perception of threat and experiencing great uncertainty. The aim of the present investigation was to determine the level of uncertainty in the family against the disease in the Shock Trauma unit, in order to contribute to the professional reflection of the nurses, to understand the different situations that the family faces and the degree of Uncertainty they face during this period. This research used the quantitative, descriptive and crosscutting approach. It had a population of 634 and a sample of 239 people. In the research was used as a data collection instrument a survey that takes as reference the Miscelessment Scale of Mishel, using the technique of non-probabilistic sampling consecutive, obtaining as a result that $68 \%$ of the family members had a regular level Of uncertainty, $26 \%$ of the population had a low level of uncertainty, and $6 \%$ had a high level of uncertainty. Finally, it was concluded that a lower level of education and shorter residence time generates a greater index of uncertainty in the relative to the disease.

\section{Introducción}

En la actualidad los servicios de Emergencia de todo el país, tanto del Seguro social de salud (ESSALUD) como del Ministerio de Salud (MINSA), se ven congestionados de personas que presentan diferentes problemas de salud. Debido a la inestabilidad del paciente, el profesional enfermero centra su atención observando y monitorizando su cuidado, de forma que, aunque conocen los requerimientos de la familia, la realidad es que pasan a un segundo e incluso a un tercer plano. El ingreso en todos los establecimientos de salud, se puede realizar de dos formas, una por medio de la consulta externa y la otra haciendo uso de los servicios de emergencia; este último acceso constituye un suceso vital, muchas veces estresante tanto para el paciente como para las personas más allegada a este, siendo su familiar en muchas ocasiones; estas vivencias generalmente son negativas ya que no sólo es debida a un ingreso por emergencia, sobre todo a la Unidad Shock Trauma, área más crítica donde se encuentran los pacientes que 
tienen alterada su hemodinámica. Si no también por la falta de insumos para los exámenes de ayuda de diagnóstico que se requiere, la demora en la atención, el diagnóstico de ingreso del paciente y sumando a ellos los obstáculos en la comunicación entre el personal médico, las enfermeras, con la familia y el paciente. Es por ello, que la enfermedad y el proceso de ingreso a un área crítica, originan muchas veces una percepción de amenaza; los familiares se enfrentan a situaciones difíciles, en la que predomina el estrés, las confusiones, la angustia, la preocupación, y sobre todo incertidumbre, y que ocasiona que se sientan solas.

Así mismo, la dinámica de las relaciones familiares se ven afectadas y se produce un cambio de roles, que agrava la situación de estrés; tienen dificultades para tomar decisiones, cuidarse mutuamente o solucionar problemas 1 . La percepción de los familiares está tremendamente influenciada por sus conocimientos y experiencias pasadas, sus características de personalidad y sobre todo por la inestabilidad en la hemodinámica del paciente.

Ante la problemática observada, las enfermeras, por las múltiples actividades que deben realizar refieren "¿no tengo tiempo para los pacientes y voy a tener tiempo para hablar con los familiares?", "yo atiendo a pacientes, no a familiares", "los familiares cuando se hacen presentes quieren que a todos les des explicaciones entre ellos no se comunican".

Por lo anteriormente expuesto surgió la siguiente interrogante: ¿Cuáles son los niveles de incertidumbre en el familiar frente a la enfermedad en la Unidad de Shock Trauma del Hospital Nacional Almanzor Aguinaga Asenjo?

Esta problemática se justificó porque en la actualidad, el índice de personas que hacen uso del servicio de emergencia ha venido incrementándose día a día, siendo así 3682 personas que se atienden al mes, de las cuales 166 en promedio son de unidad de shock trauma. A su vez la familia como fuente principal de apoyo se encuentra íntimamente relacionado con el personal de salud, por lo que se ve severamente afectada ante un suceso inesperado como es un ingreso por emergencia, aunado a ello el tener que esperar que algún profesional responsable de su cuidado les pueda informar acerca de su paciente; surgiéndose así la duda, desconcierto e incertidumbre en ellos. Así mismo la enfermera no solo debe preocuparse de la persona con un problema de salud sino también de la evolución de su cuidado hacia la familia, desde la primera interacción, brindando educación y apoyo emocional para que pueda afrontar el proceso de la enfermedad.

La presente investigación pretendió contribuir a la reflexión profesional de las enfermeras, con el fin de comprender las diferentes situaciones que atraviesa el familiar y el grado de incertidumbre que afrontan durante este periodo, ya que ante un ingreso intempestivo a un centro hospitalario, no solo se ve afectada la persona enferma, sino que se extiende al ámbito de las relaciones familiares. Así mismo la Ley del Trabajo del Enfermero 2 manifiesta que la atención que se brinda no sólo debe abarcar a la persona afectada en su salud sino también a la familia y comunidad; para que así se dé el cuidado holístico, la calidad de atención y de vida que merece tanto el paciente como el familiar que enfrenta esta situación.

\section{Metodología}

El presente trabajo de investigación es de tipo cuantitativo, no experimental porque sólo se observó el fenómeno tal y como sucede en su entorno natural para posteriormente analizarlo. Con un diseño de contrastación descriptivo porque sólo midió la información de manera independiente sobre la variable, además fue de corte transversal ${ }^{3}$.

Para conseguir la muestra de estudio se utilizó la fórmula de cálculo para población finita, en donde el número de elementos del universo fue obtenido entre los meses de marzo a junio del año 2013. Después de aplicar la fórmula para un nivel de confianza del $95 \%$ se obtuvo que " $n$ " equivale a 239 personas que conforman nuestra muestra poblacional. Así mismo se tuvo en cuenta criterios de selección, siendo los siguientes: 
Criterio de inclusión:

- Todas las personas mayores de 18 años, cuyos familiares directos estén hospitalizados en la unidad Shock - Trauma del Hospital Nacional Almanzor Aguinaga Asenjo.

- Primer ingreso al servicio de Emergencia del Hospital Nacional Almanzor Aguinaga Asenjo.

Criterios de exclusión:

- Familiares de pacientes con enfermedades crónicas tales como oncológicas o/y VIH SIDA.

- Familiares analfabetos.

- Familiares con trastornos y/o discapacidad mental.

- Familiares que hablen un idioma diferente al español.

En cuanto al muestreo, fue de tipo no probabilístico, que son aquellos en los que las unidades de análisis se recogen utilizando métodos en los que no interviene el azar, de modo que no es posible estimar la probabilidad que tiene cada elemento de ser incluido en la muestra y no todos los elementos tienen posibilidad de ser incluidos ${ }^{4}$. Se utilizó la técnica de muestro consecutivo, el cual consistió en reclutar a todos los individuos de la población accesible que cumplan con los criterios de selección durante el periodo de reclutamiento fijado para el estudio. El periodo de reclutamiento se fija en función del tamaño muestral deseado y del número de sujetos reclutarles cada día 5 .

La captación del familiar se dio a través de la Oficina de Admisión, quien emitió la conformidad de que era el primer ingreso de los pacientes a hacer uso de los servicios de emergencia; por lo tanto se ubicó al familiar presente en el pasadizo del servicio y/o en la sala de espera. La recolección de datos se realizó a través de la encuesta. Para formular este instrumento, se inició con el permiso de la Teórica Merle Mishel ${ }^{6}$, creadora de la Teoría de la Incertidumbre frente a la Enfermedad, a quien se le envió un correo solicitando la autorización para la modificación, adaptación y utilización de la Escala de Incertidumbre en la Enfermedad para este trabajo de investigación. Así mismo se pagó un derecho económico por el cuestionario original y se llenó el formulario requerido por la teórica "Request Form: MUIS-Adult".

Además, se recopiló información de la investigación doctoral, Adaptación transcultural de la escala de incertidumbre de M. Mishel, en personas con Insuficiencia renal crónica con tratamiento con hemodiálisis ${ }^{7}$, de la Universidad de Jaén; como también de la tesis de maestría Espiritualidad e Incertidumbre ante la enfermedad con personas con Diabetes mellitus tipo $2^{8}$, de la Universidad Nacional de Colombia, ambas fuentes fueron de ayuda para la formulación del cuestionario. Así mismo las preguntas 1, 2, 4, 5, 6, 7, 9, 12, 13 fueron tomadas de la Escala de Incertidumbre de $\mathrm{M}$. Mishel traducida por la investigación Espiritualidad e Incertidumbre ante la enfermedad con personas con Diabetes mellitus tipo 2; las preguntas 3 y 11 fueron tomadas de la Adaptación Transcultural de la escala de M. Mishel y las preguntas 8 y 10 fueron creadas.

Esta encuesta está formada por dos partes, una en donde se solicitó información básica al familiar, tal como su edad, sexo, parentesco, grado de instrucción, diagnóstico de ingreso de su familiar y tiempo de permanencia del familiar hospitalizado. En la segunda parte, encontramos la Escala Mishel de Incertidumbre en la Enfermedad forma para adulto adaptado para familiares. Este instrumento contiene 13 preguntas y en cada una de ellas la persona emite su grado de acuerdo respecto a una serie de afirmaciones relacionadas con su salud física / psicológica y el nivel de incertidumbre generado frente a las diversas situaciones de la enfermedad-tratamiento que debe enfrentar.

Como el cuestionario se realizó bajo un esquema dicotómico9, es decir, que constan de dos alternativas, a cada respuesta que va desde un SI a un NO, se le asignó un puntaje con un valor de 0 a 1 punto respectivamente. Un puntaje de 1 de cada pregunta refleja un nivel alto de incertidumbre en la pregunta correspondiente, excepto las interrogantes 3,4 y 7 donde el puntaje es invertido, porque el nivel de incertidumbre aumenta con el desconocimiento de información 
sobre su familiar. El puntaje máximo de la escala es 13 de puntos y el mínimo 1 puntos.

Para la validación del instrumento se utilizó la fórmula de Kurder Richardson ${ }^{3}$ (formula KR 20), la cual es un coeficiente que sirve para medir la fiabilidad en respuestas dicotómicas, el cual arrojó un resultado de 0.70 . Este instrumento cuenta con la siguiente puntuación:

Puntaje de corte calificación Bajo Nivel de Incertidumbre: entre 01 y 04 puntos.

Puntaje de corte calificación Regular Nivel de Incertidumbre: 05 - 09 puntos.

Puntaje de corte calificación Alto Nivel de Incertidumbre: entre 10 a 13 puntos.

En el procesamiento de análisis, una vez recogidos todos los datos necesarios a través de las encuestas, se prosiguió a ingresarlos directamente en el ordenador para su análisis. Los dos paquetes estadísticos más comunes para el almacenamiento de los datos y para los análisis estadísticos son Excel y SPSS. Ambos programas se caracterizan por ser hojas de cálculo con funciones estadísticas, que además generan gráficos, pero a diferencia del SPSS al Excel, sus funciones de análisis estadísticas y el manejo de los datos son más exhaustivos y flexibles. Para este trabajo de investigación se utilizó ambos programas.

En la introducción de datos en la hoja de cálculo, se consideró que las columnas representaran a las variables estudiadas, mientas tanto las filas contendrán los datos correspondientes a un solo sujeto. Una vez terminado esta fase, se proseguirá a la verificación de datos o limpieza de datos, con esto se buscará que los valores introducidos tengan sentido, que no falte valores o que no existan valores inverosímiles.

Como fase final, se generó gráficos a partir de los datos ingresados con el fin de poder examinarlos visualmente. De este modo, aparecerán las observaciones extremas, junto a cualquier otro patrón interesante.

\section{Resultados, análisis y discusión}

\section{GRÁFICO Nº1 01 \\ NIVELES DE INCERTIDUMBRE}

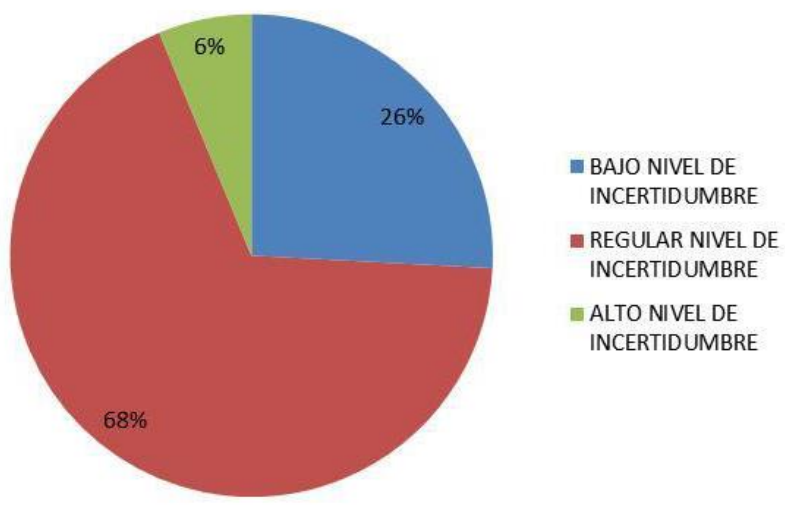

Fuente: Datos obtenidos de la recopilación de las encuestas realizadas.

Como se visualiza en el gráfico $\mathrm{N}^{\circ} 01$, el $68 \%$ de la población manifiesta un nivel regular de incertidumbre, mientras que un $26 \%$ presentan un bajo nivel de incertidumbre y tan solo un $6 \%$ percibe un alto nivel de incertidumbre.

\section{GRÁFICO N ${ }^{\circ} 02$ \\ ME PREOCUPA QUE TENGA DOLORES O SÍNTOMAS DESGRADABLES}

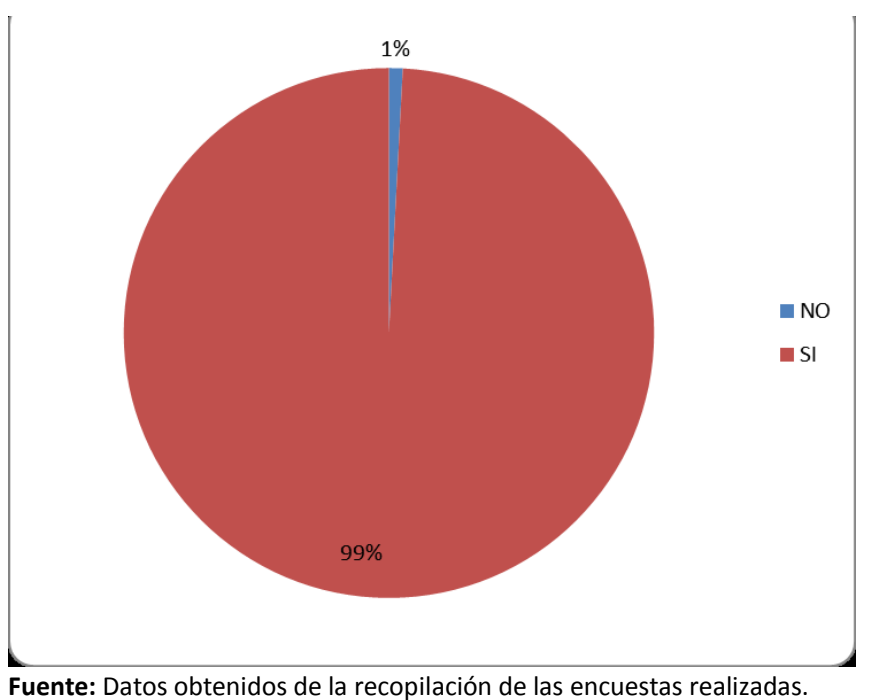

El gráfico muestra que un $99 \%$ de la población se preocupa de que su familiar tenga dolores o síntomas desagradables, mientras que el $1 \%$ comunica lo contrario. 
Esta investigación se ha determinado el nivel de Incertidumbre frente a la enfermedad; el cual es una incapacidad de determinar el significado de los hechos que tienen relación con la enfermedad ${ }^{10}$, y que afecta a las personas sin distinción alguna. En esta investigación se encontró que los familiares presentan un nivel regular de incertidumbre (68\%), nivel bajo de incertidumbre (26\%) y un nivel alto de incertidumbre (6\%); sin embargo en otras investigaciones previas realizadas en otros lugares, tiempo y servicio, se obtuvo resultados diferentes, como por ejemplo en la tesis intitulada "Creencias, Sentimientos e Incertidumbre frente a la enfermedad en familiares de pacientes ingresados a unidad de cuidados intensivos de Chile", se alcanzó como resultado que el $67 \%$ de los familiares presentó un nivel de incertidumbre alto y un $33 \%$ presentó un bajo nivel de incertidumbre.

La incertidumbre es un estado cognitivo que representa la inadecuación del esquema cognitivo existente, cuya función es la de ayudar en la interpretación de los hechos surgidos a raíz de la enfermedad ${ }^{1}$. Conocer la enfermedad del familiar y las posibles complicaciones (nos indica que existe una fuente de estructura ${ }^{12}$, en este caso la educación, que permite suplir la estructura de los eventos en el marco de estímulos ya que hay un cierto nivel de conocimientos, a los cuales le pueden dar significado y contexto, por lo tanto, va a ayudar en el afrontamiento del suceso intempestivo, como es el ingreso a la Unidad Shock Trauma ${ }^{13}$, unidad más crítica de todo el servicio de emergencia, y las complicaciones de la enfermedad en el menor tiempo posible y por lo tanto disminuye el nivel de incertidumbre, percibiendo así menor complejidad relacionada con los sucesos y también las posibles complicaciones. Sin embargo las personas que desconocen ya sea acerca de la enfermedad y/o complicaciones experimentan incertidumbre por periodos más largos. Al evaluar la tipología de síntomas se tiene en cuenta el número, frecuencia, duración y localización ${ }^{10}$, así como también el patrón que exista entre ellos, de tal forma que al haber menos ambigüedad en los síntomas, será menor el nivel de incertidumbre, por el contrario, un repentino cambio de ellos refleja su inconsistencia y no pueden ser utilizados para medir confiabilidad sobre el estado de la enfermedad, generando así incertidumbre.

En cuanto a los cuidados y el tratamiento, los familiares perciben menor complejidad en ellos cuando el agente de salud tiene una buena e importante relación con el familiar para la prevención de la incertidumbre; esto quiere decir que el agente de salud (enfermera) actúa como una fuente de estructura con el apoyo social ${ }^{12}$, ya que le da la facultad de poder retroalimentar, aclarar dudas a los familiares de acuerdo a eventos presentes; de tal forma que ayuda a las personas a generar un nuevo esquema cognitivo. Así mismo saber si los cuidados y tratamiento están dando resultados permite evidenciar la congruencia entre lo esperado y lo experimentado; no obstante, cuando no se encuentran satisfechos, el familiar mantiene la impredecibilidad e inestabilidad con el suceso ya que no obtienen los resultados esperados.

Asimismo, cuando los familiares afirman que no entienden para qué y por qué está recibiendo ese tratamiento y consideran que los cuidados y el tratamiento son difíciles de entender, es porque ellos no intentan comprender el mecanismo técnico de la enfermedad, tratamiento o cuidados; sino que requieren que el personal de salud les brinde la información de forma apropiada para ellos, es por eso que cuando se estudió los resultados obtenidos, dos de ellos mostraron relevancia ya que el familiar considera que a pesar de que estén al tanto de la enfermedad de su familiar no les pareció suficientes las explicaciones y orientaciones que se les brindó, debido a que dichas explicaciones eran demasiado técnicas y poco apropiadas, ya que tan solo lo único que le interesa al familiar es saber si el problema que se suscita tendrá solución, complicación o secuela y más que eso, desean en el fondo el apoyo emocional por parte del personal de salud para poder sobrellevar el proceso de adaptación. De 
igual forma cuando las familiares consideran que tanto los médicos y enfermeras no utilizan un lenguaje sencillo es porque reciben una información muy técnica, de tal forma que no es la más adecuada para ellos.

Por otro lado, la autoridad con credibilidad que manifiesta el personal de salud, es el grado de veracidad y confidencialidad ${ }^{2}$ que tienen los familiares con el personal de salud ya que influencia de forma positiva sobre el marco de estímulos al proporcionar información, en base a conocimientos y experiencias, mejorando así la congruencia con el evento. Aunado a ello, la enfermera trasmite seguridad al familiar y de esta manera disminuye cualquier percepción de amenaza. Por último, la información acerca del ambiente capacita a la persona para medir comportamientos esperados en diferentes circunstancias, la familiaridad con el evento previene la incertidumbre; pero en el ambiente de cuidado de la salud, la novedad y complejidad, impiden desarrollar familiaridad con los eventos ${ }^{2}$. Esta novedad ocasiona elevados niveles de incertidumbre en los familiares de pacientes que son admitidos súbitamente a alguna unidad crítica como es Shock Trauma, además de desconocer el tiempo que permanecerá su familiar ahí, generando mayores niveles de incertidumbre.

\section{Conclusiones}

En este estudio se obtuvo como resultado que un $68 \%$ presenta un nivel regular de incertidumbre, el $26 \%$ un nivel bajo de incertidumbre y el $6 \%$ un nivel alto de incertidumbre, esto se encuentra relacionado con el nivel de instrucción del familiar, el tiempo de permanencia y la información brindada en la Unidad Shock Trauma porque a menor grado de instrucción y menor tiempo de permanencia, mayor es la incertidumbre que se genera en el familiar.

Es importante la presencia del personal de salud en la prevención de la incertidumbre, es por eso que en la pregunta "Veo que el personal a cargo de su tratamiento sabe lo que hace $\mathrm{y} / \mathrm{o}$ está capacitado" existe una respuesta afirmativa del $85 \%$ de la población, de igual forma en la pregunta "Se quién es el responsable de la atención que se le brinda a mi familiar" con $91 \%$, revela que existe una autoridad con credibilidad que transmite seguridad al familiar y así disminuye la percepción de amenaza que estos puedan tener. Sin embargo en la pregunta "La actitud de los médicos y enfermeras me proporciona seguridad" evidencia una respuesta afirmativa del $52 \%$ y negativa del $48 \%$, manifestando así que una parte considerable de la población tiende a elevar su nivel de incertidumbre.

\section{Bibliografía}

1. Brevis I, Silva P. Creencias, Sentimientos e Incertidumbre frente a la enfermedad en familiares de pacientes ingresados. Revista Chilena de Medicina Intensiva. 2011; 26(1): 27-34

2. Colegio de Enfermeros del Perú. Ley del Trabajo de la Enfermera(o) No 27669. Lima: Colegio de Enfermeros del Perú; 2002.

3. Hernández R, Fernández C, Baptista M. Metodología de la investigación.5ta ed. México: McGraw Hill; 2010

4. Malave N. Trabajo modelo para enfoques de investigación acción participativa programas nacionales de formación: Escala tipo Likert; Maturin, 2007.

5. Torres CM. Adaptación transcultural de la escala Incertidumbre de M. Mishel, personas con Insuficiencia renal crónica con tratamiento con hemodiálisis [Tesis doctoral]. España: Universidad de Jaén; 2013

6. Hueso A, Cascant MJ. Metodología y Técnicas Cuantitativas de Investigación. Valencia: Universidad Politécnica de Valencia; 2012.

7. Marriner A, Raile M. Modelos y teorías en Enfermería. 6ta ed. Barcelona: Elsevier; 2007.

8. Gómez IP. Espiritualidad e Incertidumbre ante la enfermedad en persona con 
Diabetes mellitus tipo 2. Colombia: Universidad Nacional de Colombia; 2010.

9. Muria J. Gol R. Preparación, tabulación y análisis de encuestas para directivos. Madrid: ESIC EDITORIAL, 1998.

10. Alves JL, Castro CS, Ribeiro HI, Faustino HR. La incertidumbre en la enfermedad y la motivación para el tratamiento en diabéticos tipo 2. Revista Ciencia y Enfermería (Brasil). 2007; 15(4).

11. Folkman S, Schaefer C, Lazarus RS. Cognitive processes as mediators of stress and coping. In: V. Hamilton \& D.M. Warburton (Eds). Human stress and cognition: An information - processing approach. London: Wiley; 1979.

12. Gallo A, Jaraba T, Perez R, Barrios Z, Díaz A. Acompañamiento del enfermero(a) a familiares con pacientes en etapa terminal. Aplicación de la Teoría de Incertidumbre (Merle Mishel). Clínica Madre Bernarda. Cartagena: Corporación Universitaria Rafael Núñez; 2012.

13. Ministerio de Salud. Normas técnicas para proyectos de arquitectura y equipamiento de las unidades de emergencia. Perú: Perú: Ministerio de Salud; 2000. 